ESAIM: COCV 18 (2012) 915-929

DOI: $10.1051 / \mathrm{cocv} / 2011188$
ESAIM: Control, Optimisation and Calculus of Variations

www.esaim-cocv.org

\title{
DETERMINISTIC CHARACTERIZATION OF VIABILITY FOR STOCHASTIC DIFFERENTIAL EQUATION DRIVEN BY FRACTIONAL BROWNIAN MOTION*,**
}

\author{
TIANYANG NiE ${ }^{1,2}$ AND AUREL RĂŞCANU ${ }^{2,3}$
}

\begin{abstract}
In this paper, using direct and inverse images for fractional stochastic tangent sets, we establish the deterministic necessary and sufficient conditions which control that the solution of a given stochastic differential equation driven by the fractional Brownian motion evolves in some particular sets $K$. As a consequence, a comparison theorem is obtained.
\end{abstract}

Mathematics Subject Classification. 60H10, 60H20, 60G22.

Received February 8, 2011. Revised July 17, 2011

Published online November 22, 2011.

\section{INTRODUCTION}

The viability (weakly invariant) problem of a set $K$ for a differential equation is to find necessary and sufficient conditions such that for any starting point in $K$, the differential equation has at least one solution evolving in $K$. If all solutions of the differential equation satisfy the $K$-viable property, we call that the subset is invariant (strong invariant) for the equation.

Nagumo gave a criterion of the viability in terms of contingent sets in 1942 . The Nagumo theorem states that: if $f: K=\bar{K} \subset \mathbb{R}^{m} \rightarrow \mathbb{R}^{m}$ is a bounded and continuous function, then $K$ is viable for the differential equation

$$
x^{\prime}(t)=f(x(t)), \quad x(0)=x_{0} \in K
$$

if and only if, $\forall x \in K$ and $\forall p$, a normal vector at $K$ in $x$, we have

$$
\langle f(x), p\rangle \leq 0 .
$$

The important tools in studying the viability problem are the notions of tangent sets and contingent sets. Aubin and Da Prato firstly used the stochastic contingent sets to characterize the viability and invariance for

\footnotetext{
Keywords and phrases. Stochastic viability, stochastic differential equation, stochastic tangent set, fractional Brownian motion.

* Supported by Marie Curie ITN Project, "Controlled Systems", No. 213841/2008.

** Supported by IDEI project, No. 395/200\%.

1 School of Mathematics, Shandong University, Jinan, Shandong 250100, P.R. China. nietianyang@163.com; tianyang.nie@uaic.ro

2 Faculty of Mathematics, "Alexandru Ioan Cuza" University, Carol I Blvd, No. 11, 700506 Iasi, Romania. aurel.rascanu@uaic.ro

3 "Octav Mayer" Mathematics Institute of the Romanian Academy, Carol I Blvd, No. 8, 700506 Iasi, Romania.
} 
the stochastic differential equations (SDEs) of a closed and convex subset in [1]. Milian generalized Aubin and Da Prato's result to arbitrary subsets which can be time-dependent and random in [9].

Another approach was developed by Buckdahn et al. in [3-6]. The main points of their work consist in proving that the viability property for SDE and also backward SDE holds true if and only if the square of the distance to the constraint sets is a viscosity supersolution (subsolution) of the associated Hamilton-Jacobi-Bellman equation.

A general result on the existence and uniqueness of the solution for multidimensional, time dependent, SDEs driven by fBm with Hurst parameter $H>1 / 2$ was given by Nualart and Rascanu in [11], using the techniques of the classical fractional calculus. Moreover, Ciotir and Rascanu proved a type of Nagumo Theorem on viability property of closed bounded subsets for the SDE driven by fBm in [7].

Criterions for the viability and invariance of closed bounded subsets expressed by stochastic contingent sets given in [7] are general, but their conditions are very hard to check, even for the particular case. Using the ideas of [7], our paper will give easier checkable conditions for general SDEs driven by fBm for some particular sets $K$. As a very important application of our results, a comparison theorem for the SDEs driven by fBm is obtained.

In fact, from [7], we give the extension of the result concerning stochastic tangent sets to direct images and inverse images which was studied by Aubin and Da Prato in [1], to the following stochastic differential equation driven by fractional Brownian motion, $\mathbb{P}$-a.s. $\omega \in \Omega$,

$$
X_{s}^{t, x}=x+\int_{t}^{s} b\left(r, X_{r}^{t, x}\right) \mathrm{d} r+\int_{t}^{s} \sigma\left(r, X_{r}^{t, x}\right) \mathrm{d} B_{r}^{H}, \quad s \in[t, T]
$$

where

- $B^{H}=\left\{B_{t}^{H}, t \geq 0\right\}$ is a $k$-dimensional $\mathrm{fBm}$ with Hurst parameter $1 / 2<H<1$, and the integral with respect to $B^{H}$ is a pathwise Riemann-Stieltjes integral;

- $b(t, x):[0, T] \times \mathbb{R}^{d} \rightarrow \mathbb{R}^{d}$ and $\sigma(t, x):[0, T] \times \mathbb{R}^{d} \rightarrow \mathbb{R}^{d \times k}$ are continuous functions.

In the case of one-dimensional $\mathrm{fBm}$, we derive explicit criteria for the solution of this equation to remain in some particular sets $K$ i.e., under which it holds that for all $t \in[0, T]$ and for all $x \in K$,

$$
X_{s}^{t, x} \in K \quad \text { a.s. } \omega \in \Omega, \quad \forall s \in[t, T] .
$$

Similarly to [1], the characterization of viability of $K$ is obtained through the study of the direct and inverse images for fractional stochastic tangent sets. Using our main Theorem 3.5, we get the conditions on $b$ and $\sigma$ such that some particular sets $K$ are viable.

Now we explain how the paper is organized. In the second section, we recall some classical definitions and assumptions on the coefficients. We also recall the main results from [7], which we will use later. In Section 3, we introduce the notion of direct and inverse images corresponding to SDE driven by fBm and give our main results. Section 4 is devoted to give the deterministic characterization of viability conditions for some particular set constraints. In Section 5, as an application, a comparison theorem is proved.

\section{Preliminaries}

Consider the following stochastic equation on $\mathbb{R}^{d}$

$$
X_{s}=X_{0}+\int_{0}^{s} b\left(r, X_{r}\right) \mathrm{d} r+\int_{0}^{s} \sigma\left(r, X_{r}\right) \mathrm{d} B_{r}^{H}, \quad s \in[0, T]
$$

where,

- $B^{H}=\left\{B_{t}^{H}, t \geq 0\right\}$ is a $k$-dimensional $\mathrm{fBm}$ defined on a complete probability space $(\Omega, \mathcal{F}, \mathbb{P})$, with Hurst parameter $1 / 2<H<1$, and the integral with respect to $B^{H}$ is a pathwise Riemann-Stieltjes integral; 
- $X_{0}$ is a $d$-dimensional random variable;

- $b:[0, T] \times \mathbb{R}^{d} \rightarrow \mathbb{R}^{d}, \sigma:[0, T] \times \mathbb{R}^{d} \rightarrow \mathbb{R}^{d \times k}$ are continuous functions.

Remark 2.1. The fractional Brownian motion has the following properties:

For every $0<\varepsilon<H$ and $T>0$, there exists a positive random variable $\eta_{\varepsilon, T}$ such that $\mathbb{E}\left(\left|\eta_{\varepsilon, T}\right|^{p}\right)$ $<\infty$, for all $p \in[1, \infty)$ and for all $s, t \in[0, T]$,

$$
\left|B^{H}(t)-B^{H}(s)\right| \leq \eta_{\varepsilon, T}|t-s|^{H-\varepsilon}, \quad \text { a.s. }
$$

From Proposition 1.7.1, [2] (see also [8]), we know that for every $t_{0} \in[0,+\infty)$,

$$
\mathbb{P}\left\{\limsup _{\substack{t \rightarrow t_{0}, t \geq t_{0}}}\left|\frac{B^{H}(t)-B^{H}\left(t_{0}\right)}{t-t_{0}}\right|=+\infty\right\}=1
$$

Using the same method, we can prove that

$$
\mathbb{P}\left\{\limsup _{\substack{t \rightarrow t_{0}, t \geq t_{0}}} \frac{B^{H}(t)-B^{H}\left(t_{0}\right)}{t-t_{0}}=+\infty\right\}=\mathbb{P}\left\{\liminf _{\substack{t \rightarrow t_{0}, t \geq t_{0}}} \frac{B^{H}(t)-B^{H}\left(t_{0}\right)}{t-t_{0}}=-\infty\right\}=\frac{1}{2} .
$$

\subsection{Assumptions and notations}

For the coefficients appearing in equation (2.1), we make the following assumptions (as in [7]):

$\left(\mathbf{H}_{1}\right) \sigma(t, x)$ is differentiable with respect to $x$, and there exist $\beta, \delta, M_{0}, 0<\beta, \delta \leq 1$ and, for $\forall R>0$, there exists a constant $M_{R}>0$ such that for all $t \in[0, T]$,

$$
\left(H_{\sigma}\right): \begin{cases}\text { (i) }|\sigma(t, x)-\sigma(s, y)| \leq M_{0}\left(|t-s|^{\beta}+|x-y|\right), & \forall x, y \in \mathbb{R}^{d}, \\ \text { (ii) }\left|\nabla_{x} \sigma(t, y)-\nabla_{x} \sigma(s, z)\right| \leq M_{R}\left(|t-s|^{\beta}+|y-z|^{\delta}\right), & \forall|y|,|z| \leq R,\end{cases}
$$

where $\nabla_{x} \sigma(t, x)=\left(\nabla_{x} \sigma^{i, j}(t, x)\right)_{i=\overline{1, d}, j=\overline{1, k}}$ and

$$
\left|\nabla_{x} \sigma(t, x)\right|^{2}=\sum_{l=1}^{d} \sum_{i=1}^{d} \sum_{j=1}^{k}\left|\partial_{x_{l}} \sigma^{i, j}(t, x)\right|^{2} .
$$

From (i), we can deduce that for all $x \in \mathbb{R}^{d}$

$$
|\sigma(t, x)| \leq|\sigma(0,0)|+M_{0}\left(|t|^{\beta}+|x|\right) \leq M_{0, T}(1+|x|),
$$

where $M_{0, T}=|\sigma(0,0)|+M_{0}+M_{0} T$.

Let

$$
\alpha_{0}=\min \left\{\beta, \frac{\delta}{1+\delta}\right\}
$$

$\left(\mathbf{H}_{2}\right)$ There exist $\mu \in\left(1-\alpha_{0}, 1\right], L_{0}$ and, for $\forall R \geq 0$, there exists a constant $L_{R}>0$ such that $\forall t \in[0, T]$,

$$
\left(H_{b}\right):\left\{\begin{array}{l}
(\mathrm{i})|b(r, x)-b(s, y)| \leq L_{R}\left(|r-s|^{\mu}+|x-y|\right), \quad \forall|x|,|y| \leq R, \\
\text { (ii) }|b(t, x)| \leq L_{0}(1+|x|), \quad \forall x \in \mathbb{R}^{d} .
\end{array}\right.
$$

Finally, we introduce some notations. 
Given $A=\left(a^{i, j}\right)_{d \times k}$ and $y=\left(y^{i}\right)_{d \times 1}, d, k \in \mathbb{N}^{*}$. we denote $|A|^{2}=\sum_{i, j}\left|a^{i, j}\right|^{2}$ and $|y|=\sum_{i}\left|y^{i}\right|^{2}$. Let $t \in[0, T]$ be fixed. Denote by

- $W^{\alpha, \infty}\left(t, T ; \mathbb{R}^{d}\right), 0<\alpha<1$, the space of functions $f:[t, T] \rightarrow \mathbb{R}^{d}$ which are continuous and

$$
\|f\|_{\alpha, \infty ;[t, T]}:=\sup _{s \in[t, T]}\left(|f(s)|+\int_{t}^{s} \frac{|f(s)-f(r)|}{(s-r)^{\alpha+1}} \mathrm{~d} r\right)<\infty .
$$

- $\tilde{W}^{1-\alpha, \infty}\left(t, T ; \mathbb{R}^{d}\right), 0<\alpha<1 / 2$, the space of continuous functions $g:[t, T] \rightarrow \mathbb{R}^{d}$ such that

$$
\|g\|_{\tilde{W}^{1-\alpha, \infty}\left(t, T ; \mathbb{R}^{d}\right)}:=|g(t)|+\sup _{t<r<s<T}\left(\frac{|g(s)-g(r)|}{(s-r)^{1-\alpha}}+\int_{r}^{s} \frac{|g(y)-g(r)|}{(y-r)^{2-\alpha}} \mathrm{d} y\right)<\infty .
$$

- $C^{\mu}\left([t, T] ; \mathbb{R}^{d}\right), 0<\mu<1$, the space of $\mu$-Hölder continuous functions $f:[t, T] \rightarrow \mathbb{R}^{d}$, with the norm

$$
\|f\|_{\mu ;[t, T]}=\sup _{s \in[t, T]}|f(s)|+\sup _{t \leq r<s \leq T} \frac{|f(s)-f(r)|}{(s-r)^{\mu}}<\infty .
$$

- $W^{\alpha, 1}\left(t, T ; \mathbb{R}^{d}\right)$ the space of measurable functions $f$ such that

$$
\|f\|_{\alpha, 1 ;[t, T]}:=\int_{t}^{T}\left[\frac{|f(s)|}{(s-t)^{\alpha}}+\int_{t}^{s} \frac{|f(s)-f(y)|}{(s-y)^{\alpha+1}} \mathrm{~d} y\right] \mathrm{d} s<\infty,
$$

and we have

$$
W^{\alpha, \infty}\left(t, T ; \mathbb{R}^{d}\right) \subset W^{\alpha, 1}\left(t, T ; \mathbb{R}^{d}\right) .
$$

\subsection{Generalized Stieltjes integral}

Denoting

$$
\Lambda_{\alpha}(g ;[t, T]):=\frac{1}{\Gamma(1-\alpha)} \sup _{t<r<s<T}\left|\left(D_{s-}^{1-\alpha} g_{s-}\right)(r)\right|
$$

where

$$
\Gamma(\alpha)=\int_{0}^{\infty} s^{\alpha-1} \mathrm{e}^{-s} \mathrm{~d} s
$$

and

$$
\left(D_{s-}^{1-\alpha} g_{s-}\right)(r)=\frac{\mathrm{e}^{\mathrm{i} \pi(1-\alpha)}}{\Gamma(\alpha)}\left(\frac{g(s)-g(r)}{(s-r)^{1-\alpha}}+(1-\alpha) \int_{r}^{s} \frac{g(r)-g(y)}{(y-r)^{2-\alpha}} \mathrm{d} y\right) 1_{(t, s)}(r),
$$

it follows that (see $[10,11])$

$$
\Lambda_{\alpha}(g ;[t, T]) \leq \frac{1}{\Gamma(1-\alpha) \Gamma(\alpha)}\|g\|_{\tilde{W}^{1-\alpha, \infty}\left(t, T ; \mathbb{R}^{d}\right)} .
$$

Also it's obvious that

$$
\Lambda_{\alpha}(g ;[t, T]) \leq \Lambda_{\alpha}(g ;[0, T]) \quad\left(:=\Lambda_{\alpha}(g)\right) .
$$

Definition 2.2. Let $0<\alpha<1 / 2$. We assume $f \in W^{\alpha, 1}\left(t, T ; \mathbb{R}^{d \times k}\right)$ and $g \in \tilde{W}^{1-\alpha, \infty}\left(t, T ; \mathbb{R}^{k}\right)$, then the generalized Stieltjes integral is defined by

$$
\int_{t}^{s} f(r) \mathrm{d} g(r):=(-1)^{\alpha} \int_{t}^{s}\left(D_{t+}^{\alpha} f\right)(r)\left(D_{s-}^{1-\alpha} g_{s-}\right)(r) \mathrm{d} r
$$


where

$$
\left(D_{t+}^{\alpha} f\right)(r)=\frac{1}{\Gamma(1-\alpha)}\left(\frac{f(r)}{(r-t)^{\alpha}}+\alpha \int_{t}^{r} \frac{f(r)-f(y)}{(r-y)^{\alpha+1}} \mathrm{~d} y\right) 1_{(t, T)}(r) .
$$

The integral $\int_{t}^{s} f(r) \mathrm{d} g(r)$ exists for all $s \in[t, T]$ and

$$
\begin{aligned}
\left|\int_{t}^{T} f(r) \mathrm{d} g(r)\right| & \leq \sup _{t \leq r<s \leq T}\left|\left(D_{s-}^{1-\alpha} g_{s-}\right)(r)\right| \int_{t}^{T}\left|\left(D_{t+}^{\alpha} f\right)(s) \mathrm{d} s\right| \\
& \leq \Lambda_{\alpha}(g ;[t, T])\|f\|_{\alpha, 1 ;[t, T] .}
\end{aligned}
$$

Remark 2.3. It is known that when $H \in(1 / 2,1)$ and $1-H<\alpha<1 / 2$, the random variable

$$
G=\Lambda_{\alpha}\left(B^{H}\right)=\frac{1}{\Gamma(1-\alpha)} \sup _{t<s<r<T}\left|\left(D_{r-}^{1-\alpha} B_{r-}\right)(s)\right|
$$

has moments of all order. Therefore if $u=\left\{u_{t}, t \in[0, T]\right\}$ is a stochastic process and its trajectories belong to $W^{\alpha, 1}\left(t, T ; \mathbb{R}^{d}\right)$, with $1-H<\alpha<1 / 2$, the integral $\int_{0}^{T} u_{s} \mathrm{~d} B_{s}^{H}$ has the meaning in the sense of Definition 2.2 and it follows

$$
\left|\int_{0}^{T} u_{s} \mathrm{~d} B_{s}^{H}\right| \leq G\|u\|_{\alpha, 1},
$$

which means that the stochastic integral from (2.1) is a pathwise Riemann-Stieltjes integral.

Nualart and Rascanu proved in [11] that under the assumptions $\left(\mathbf{H}_{1}\right)$ and $\left(\mathbf{H}_{2}\right)$, with $\beta>1-H$ and $\delta>1 / H-1$, the SDE

$$
X_{s}^{t, \xi}=\xi+\int_{t}^{s} b\left(r, X_{r}^{t, \xi}\right) \mathrm{d} r+\int_{t}^{s} \sigma\left(r, X_{r}^{t, \xi}\right) \mathrm{d} B_{r}^{H}, s \in[t, T]
$$

has a unique solution $X^{t, \xi} \in L^{0}\left(\Omega, \mathcal{F}, \mathbb{P} ; W^{\alpha, \infty}\left(t, T ; \mathbb{R}^{d}\right)\right)$, for all $\alpha \in\left(1-H, \alpha_{0}\right)$. Moreover, for $\mathbb{P}$-almost all $\omega \in \Omega, X^{t, \xi}(\omega) \in C^{1-\alpha}\left(0, T ; \mathbb{R}^{d}\right)$.

\subsection{Fractional viability}

Following [7], we recall the notion of the viability property for SDE driven by fBm. We also present the characterization theorem for the viability property.

Consider the stochastic differential equation driven by $\mathrm{fBm} B^{H}$ with Hurst parameter $1 / 2<H<1$,

$$
X_{s}^{t, x}=x+\int_{t}^{s} b\left(r, X_{r}^{t, x}\right) \mathrm{d} r+\int_{t}^{s} \sigma\left(r, X_{r}^{t, x}\right) \mathrm{d} B_{r}^{H}, \quad s \in[t, T]
$$

Definition 2.4. Let $\mathcal{K}=\{K(t): t \in[0, T]\}$ be a family of subsets of $\mathbb{R}^{d}$. We say that $\mathcal{K}$ is viable (weakly invariant) for equation (2.4) if, for every $t \in[0, T]$ and from every starting point $x \in K(t)$, there exists at least one of its solutions $\left\{X_{s}^{t, x}: s \in[t, T]\right\}$ which satisfies

$$
X_{s}^{t, x}(\omega) \in K(s) \text { for all } s \in[t, T], \quad \text { a.s. } \omega \in \Omega .
$$

Definition 2.5. The family $\mathcal{K}$ is invariant (strong invariant) for equation (2.4) if, starting at every time $t \in[0, T]$ and from every point $x \in K(t)$, all solutions $\left\{X_{s}^{t, x}: s \in[t, T]\right\}$ of (2.4) satisfy

$$
X_{s}^{t, x}(\omega) \in K(s) \text { for all } s \in[t, T], \quad \text { a.s. } \omega \in \Omega .
$$


Remark 2.6. In the case that the equation has a unique solution, viability is equivalent to invariance.

Suppose that the mappings $b$ and $\sigma$ satisfy $\left(\mathbf{H}_{1}\right)$ and $\left(\mathbf{H}_{2}\right)$.

Definition 2.7. Let $t \in[0, T]$ and $x \in K(t)$. Let $1 / 2<1-\alpha<H$.

The $(1-\alpha)$-fractional $B^{H}$-contingent set to $K(t)$ in $x$ is the set of the pairs $(u, v) \in \mathbb{R}^{d} \times \mathbb{R}^{d \times k}$, such that there exist a random variable $\bar{h}=\bar{h}^{t, x}>0$ and a stochastic process $Q=Q^{t, x}: \Omega \times[t, t+\bar{h}] \rightarrow \mathbb{R}^{d}$, and for any $R>0$ with $|x| \leq R$, there exist two random variables $H_{R}, \tilde{H}_{R}>0$, depending only on $R, L_{R}, M_{0, T}, M_{0}, L_{0}$, $\left.T, \alpha, \beta, \Lambda_{\alpha}\left(B^{H}\right)\right)$ and a constant $\gamma=\gamma_{R}(\alpha, \beta) \in(0,1)$ such that for all $s, \tau \in[t, t+\bar{h}], \mathbb{P}$-a.s.

$$
|Q(s)-Q(\tau)| \leq H_{R}|s-\tau|^{1-\alpha}, \quad|Q(s)| \leq \tilde{H}_{R}|s-t|^{1+\gamma}
$$

and

$$
x+(s-t) u+v\left[B_{s}^{H}-B_{t}^{H}\right]+Q(s) \in K(s) .
$$

Definition 2.8. Let $t \in[0, T]$ and $x \in K(t)$. Let $1 / 2<1-\alpha<H$.

The $(1-\alpha)$-fractional $B^{H}$-tangent set to $K(t)$ in $x$, denoted by $S_{K(t)}(t, x)$, is the set of the pairs $(u, v) \in$ $\mathbb{R}^{d} \times \mathbb{R}^{d \times k}$, such that there exist a random variable $\bar{h}=\bar{h}^{t, x}>0$ and two stochastic processes

$$
\begin{aligned}
& U=U^{t, x}: \Omega \times[t, T] \rightarrow \mathbb{R}^{d}, \quad U(t)=0, \\
& V=V^{t, x}: \Omega \times[t, T] \rightarrow \mathbb{R}^{d \times k}, \quad V(t)=0
\end{aligned}
$$

and for every $R>0$ with $|x| \leq R$, there exist two random variables $D_{R}, \tilde{D}_{R}>0$, depending only on $R, L_{R}$, $\left.M_{0, T}, M_{0}, L_{0}, T, \alpha, \beta, \Lambda_{\alpha}\left(B^{H}\right)\right)$, such that for all $s, \tau \in[t, t+\bar{h}], \mathbb{P}$-a.s.

$$
|U(s)-U(\tau)| \leq D_{R}|s-\tau|^{1-\alpha}, \quad|V(s)-V(\tau)| \leq \tilde{D}_{R}|s-\tau|^{\min \{\beta, 1-\alpha\}}
$$

and

$$
x+\int_{t}^{s}(u+U(r)) \mathrm{d} r+\int_{t}^{s}(v+V(r)) \mathrm{d} B_{r}^{H} \in K(s) .
$$

Remark 2.9. The definition of $S_{\varphi(K(t))}(t, \varphi(x))$ is similar to $S_{K(t)}(t, x)$, we only change the condition

$$
x+\int_{t}^{s}(u+U(r)) \mathrm{d} r+\int_{t}^{s}(v+V(r)) \mathrm{d} B_{r}^{H} \in K(s),
$$

to the following form

$$
\varphi(x)+\int_{t}^{s}(u+U(r)) \mathrm{d} r+\int_{t}^{s}(v+V(r)) \mathrm{d} B_{r}^{H} \in \varphi(K(s)) .
$$

Now we recall the main result of [7] concerning the stochastic viability.

Theorem 2.10. Let $\mathcal{K}=\{K(t): t \in[0, T]\}, K(t)=\overline{K(t)} \subset \mathbb{R}^{d}$. Assume that $\left(\mathbf{H}_{1}\right)$ and $\left(\mathbf{H}_{2}\right)$ are satisfied with $1 / 2<H<1,1-H<\beta, \delta>\frac{1-H}{H}$. Let $\max \{1-H, 1-\mu\}<\alpha<\alpha_{0}$. Then the following assertions are equivalent:

$(I) \mathcal{K}$ is viable for the fractional $S D E(2.4)$, i.e., for all $t \in[0, T]$ and for all $x \in K(t)$ there exists a solution $X^{t, x}(\omega, \cdot) \in C^{1-\alpha}\left([t, T] ; \mathbb{R}^{d}\right)$ of $(2.4)$ such that

$$
X_{s}^{t, x} \in K(s), \quad \forall s \in[t, T] ;
$$

(II) for all $t \in[0, T], x \in K(t),(b(t, x), \sigma(t, x))$ is $(1-\alpha)$-fractional $B^{H}$-contingent to $K(t)$ in $x$;

(III) for all $t \in[0, T], x \in K(t),(b(t, x), \sigma(t, x))$ is $(1-\alpha)$-fractional $B^{H}$-tangent to $K(t)$ in $x$. 
Remark 2.11. The assertion $(I I I)$ is given in [7] only for the deterministic case, i.e. using $g \in \tilde{W}^{1-\alpha, \infty}\left(t, T ; \mathbb{R}^{d}\right)$ instead of $B^{H}$. Using the same method as in [7] to prove $(I I)$, we can derive $(I I I)$.

Moreover, it follows:

Corollary 2.12. Assume that $\left(\mathbf{H}_{1}\right)$ and $\left(\mathbf{H}_{2}\right)$ are satisfied with $1 / 2<H<1,1-H<\beta, \delta>\frac{1-H}{H}$. Let $\max \{1-H, 1-\mu\}<\alpha<\alpha_{0}$, if $K \subset \mathbb{R}^{d}$ is independent of $t$, the following assertions are equivalent:

(j) $K$ is viable with respect to the fractional $S D E(2.4)$;

(jj) for all $t \in[0, T], x \in \partial K,(b(t, x), \sigma(t, x))$ is $(1-\alpha)$-fractional $B^{H}$-contingent to $K$ in $x$;

(jjj) for all $t \in[0, T], x \in \partial K,(b(t, x), \sigma(t, x))$ is $(1-\alpha)$-fractional $B^{H}$-tangent to $K$ in $x$.

Proof. Since $K$ is independent of $t$, from Theorem 2.10, it's obvious that $(j) \Rightarrow(j j) \Rightarrow(j j j)$. It is sufficient to prove $(j j j) \Rightarrow(j)$.

Let $t \in[0, T]$ and $x \in K \backslash \partial K$ be chosen arbitrary. Since $X^{t, x}$ is continuous, there exists a random variable $\bar{h}$, such that for all $s \in[t, t+\bar{h}]$,

$$
X_{s}^{t, x}=x+\int_{t}^{s} b\left(r, X_{r}^{t, x}\right) \mathrm{d} r+\int_{t}^{s} \sigma\left(r, X_{r}^{t, x}\right) \mathrm{d} B_{r}^{H} \in K,
$$

which can be written as

$$
X_{s}^{t, x}=x+\int_{t}^{s}[b(t, x)+U(r)] \mathrm{d} r+\int_{t}^{s}[\sigma(t, x)+V(r)] \mathrm{d} B_{r}^{H} \in K,
$$

where

$$
U(r)=b\left(r, X_{r}^{t, x}\right)-b(t, x), \quad V(r)=\sigma\left(r, X_{r}^{t, x}\right)-\sigma(t, x) .
$$

Clearly $(b(t, x), \sigma(t, x))$ is $(1-\alpha)$-fractional $B^{H}$-tangent to $K$ in $x$. From $(j j j)$, taking into account that $K$ is independent of $t$, we obtain $(I I I)$.

\section{Stochastic tangent Sets to DiRECT AND InVERSE Images}

Firstly, we give the following auxiliary lemma,

Lemma 3.1. Given two stochastic processes $U=U^{t, x}, V=V^{t, x}$ satisfying (2.5), (2.6). Then for all $t \leq \tau \leq$ $s \leq t+\bar{h}$,

$$
\begin{aligned}
& \text { (a) }\left|\int_{\tau}^{s} U(r) \mathrm{d} r\right| \leq D_{R}(s-t)^{1-\alpha}(s-\tau) \\
& \text { (b) }\left|\int_{\tau}^{s} V(r) \mathrm{d} B_{r}^{H}\right| \leq C_{R}(\alpha, \beta) \tilde{D}_{R} \Lambda_{\alpha}\left(B^{H}\right)(s-t)^{\min \{\beta, 1-\alpha\}}(s-\tau)^{1-\alpha}
\end{aligned}
$$

where $C_{R}(\alpha, \beta)$ depends only on $R, \alpha$, and $\beta$.

Proof. (a)

$$
\left|\int_{\tau}^{s} U(r) \mathrm{d} r\right|=\left|\int_{\tau}^{s}[U(r)-U(t)] \mathrm{d} r\right| \leq D_{R}\left|\int_{\tau}^{s}(r-t)^{1-\alpha} \mathrm{d} r\right| \leq D_{R}(s-t)^{1-\alpha}(s-\tau),
$$


(b)

$$
\begin{aligned}
\left|\int_{\tau}^{s} V(r) \mathrm{d} B_{r}^{H}\right|= & \left|\int_{\tau}^{s}[V(r)-V(t)] \mathrm{d} B_{r}^{H}\right| \\
\leq & \Lambda_{\alpha}\left(B^{H}\right) \mid V \|_{\alpha, 1 ;[\tau, s]} \\
\leq & \Lambda_{\alpha}\left(B^{H}\right) \int_{\tau}^{s}\left[\frac{|V(r)-V(t)|}{(r-\tau)^{\alpha}}+\int_{\tau}^{r} \frac{|V(r)-V(y)|}{(r-y)^{\alpha+1}} \mathrm{~d} y\right] \mathrm{d} r \\
\leq & \tilde{D}_{R} \Lambda_{\alpha}\left(B^{H}\right) \int_{\tau}^{s}\left[\frac{(r-t)^{\min \{\beta, 1-\alpha\}}}{(r-\tau)^{\alpha}}+\int_{\tau}^{r} \frac{(r-y)^{\min \{\beta, 1-\alpha\}}}{(r-y)^{\alpha+1}} \mathrm{~d} y\right] \mathrm{d} r \\
\leq & \tilde{D}_{R} \Lambda_{\alpha}\left(B^{H}\right)\left[\frac{1}{1-\alpha}(s-t)^{\min \{\beta, 1-\alpha\}}(s-\tau)^{1-\alpha}\right. \\
& \left.+\int_{\tau}^{s} \int_{\tau}^{r}(r-y)^{\min \{\beta-\alpha, 1-2 \alpha\}-1} \mathrm{~d} y \mathrm{~d} r\right] \\
\leq & C_{R}(\alpha, \beta) \tilde{D}_{R} \Lambda_{\alpha}\left(B^{H}\right)(s-t)^{\min \{\beta, 1-\alpha\}}(s-\tau)^{1-\alpha} .
\end{aligned}
$$

Remark 3.2. From $(a)$ and $(b)$, we see that for $\tau=t$,

$$
\begin{aligned}
& \left(a^{\prime}\right)\left|\int_{t}^{s} U(r) \mathrm{d} r\right| \leq D_{R}(s-t)^{2-\alpha} \\
& \left(b^{\prime}\right)\left|\int_{t}^{s} V(r) \mathrm{d} B_{r}^{H}\right| \leq C_{R}(\alpha, \beta) \tilde{D}_{R} \Lambda_{\alpha}\left(B^{H}\right)(s-t)^{1+\min \{\beta-\alpha, 1-2 \alpha\}} .
\end{aligned}
$$

The following theorem will give the extension to the fBM framework of the result concerning stochastic tangent sets to direct images which was studied by Aubin and Da Prato, [1].

Theorem 3.3. Assume that $\left(\mathbf{H}_{1}\right)$ and $\left(\mathbf{H}_{2}\right)$ are satisfied with $1 / 2<H<1,1-H<\beta, \delta>\frac{1-H}{H}$. Let $\alpha, \alpha_{0}$ be such that $\max \{1-H, 1-\mu\}<\alpha<\alpha_{0}$. Let $K(t)=\overline{K(t)} \subset \mathbb{R}^{d}, t \in[0, T], S_{K(t)}(t, x)$ be $(1-\alpha)$-fractional $B^{H}$-tangent set to $K$ in $x$ and $\varphi$ be a $C^{2}$ map from $\mathbb{R}^{d}$ to $\mathbb{R}^{m}$ with a bounded second derivative. If

$$
(b(t, x), \sigma(t, x)) \in S_{K(t)}(t, x)
$$

then

$$
\left(\varphi^{\prime}(x) b(t, x), \varphi^{\prime}(x) \sigma(t, x)\right) \in S_{\varphi(K(t))}(t, \varphi(x)) .
$$

Proof. Since $(b(t, x), \sigma(t, x)) \in S_{K(t)}(t, x)$, there exist a random variable $\bar{h}=\bar{h}^{t, x}>0$ and, for all $R>0$, two stochastic processes satisfying (2.5), (2.6) for all $s \in[t, t+\bar{h}]$, and $x \in \mathbb{R}^{d}$ with $|x| \leq R$,

$$
x+\int_{t}^{s}(b(t, x)+U(r)) \mathrm{d} r+\int_{t}^{s}(\sigma(t, x)+V(r)) \mathrm{d} B^{H}(r) \in K(s)
$$

Define

$$
\eta_{s}=x+\int_{t}^{s}(b(t, x)+U(r)) \mathrm{d} r+\int_{t}^{s}(\sigma(t, x)+V(r)) \mathrm{d} B^{H}(r) .
$$

From Lemma 3.1 and the $(H-\varepsilon)$ Hölder continuous property of fractional Brownian motion, it follows that for all $s, \tau \in[t, t+\bar{h}]$,

$$
\left|\eta_{s}-\eta_{\tau}\right| \leq \zeta(s-\tau)^{1-\alpha} .
$$


According to the fractional Itô formula (see [10], Rem. 2.7.4), we know that for all $s \in[t, t+\bar{h}]$

$$
\begin{aligned}
& \varphi\left(x+\int_{t}^{s}(b(t, x)+U(r)) \mathrm{d} r+\int_{t}^{s}(\sigma(t, x)+V(r)) \mathrm{d} B^{H}(r)\right) \\
& =\varphi(x)+\int_{t}^{s}\left[\varphi^{\prime}\left(\eta_{r}\right)(b(t, x)+U(r))\right] \mathrm{d} r+\int_{t}^{s}\left[\varphi^{\prime}\left(\eta_{r}\right)(\sigma(t, x)+V(r))\right] \mathrm{d} B^{H}(r) \\
& =\varphi(x)+\int_{t}^{s}\left[\varphi^{\prime}(x) b(t, x)+U_{1}(r)\right] \mathrm{d} r+\int_{t}^{s}\left[\varphi^{\prime}(x) \sigma(t, x)+V_{1}(r)\right] \mathrm{d} B^{H}(r),
\end{aligned}
$$

where

$$
\begin{aligned}
& U_{1}(r)=\varphi^{\prime}\left(\eta_{r}\right) U(r)+\left(\varphi^{\prime}\left(\eta_{r}\right)-\varphi^{\prime}(x)\right) b(t, x), \\
& V_{1}(r)=\varphi^{\prime}\left(\eta_{r}\right) V(r)+\left(\varphi^{\prime}\left(\eta_{r}\right)-\varphi^{\prime}(x)\right) \sigma(t, x) .
\end{aligned}
$$

Then

$$
\begin{aligned}
& \varphi(x)+\int_{t}^{s}\left[\varphi^{\prime}(x) b(t, x)+U_{1}(r)\right] \mathrm{d} r+\int_{t}^{s}\left[\varphi^{\prime}(x) \sigma(t, x)+V_{1}(r)\right] \mathrm{d} B^{H}(r) \\
= & \varphi\left(x+\int_{t}^{s}(b(t, x)+U(r)) \mathrm{d} r+\int_{t}^{s}(\sigma(t, x)+V(r)) \mathrm{d} B^{H}(r)\right) \in \varphi(K(s)),
\end{aligned}
$$

and

$$
U_{1}(t)=0, \quad V_{1}(t)=0 .
$$

For all $s, \tau \in[t, t+\bar{h}]$ and for every $R>0$ and $|x| \leq R$, using the Lipschitz continuity of $\varphi^{\prime}$ and $\left(\mathbf{H}_{2}\right)$, we obtain that

$$
\begin{aligned}
\left|U_{1}(s)-U_{1}(\tau)\right| & \leq\left|\varphi^{\prime}\left(\eta_{\tau}\right)\right||U(s)-U(\tau)|+(|U(s)|+|b(t, x)|)\left|\varphi^{\prime}\left(\eta_{s}\right)-\varphi^{\prime}\left(\eta_{\tau}\right)\right| \\
& \leq \theta_{1}|s-\tau|^{1-\alpha}+\theta_{2}\left|\eta_{s}-\eta_{\tau}\right| \leq \theta|s-\tau|^{1-\alpha}
\end{aligned}
$$

similarly we can prove that

$$
\left|V_{1}(s)-V_{1}(\tau)\right| \leq \tilde{\theta}|s-\tau|^{\min \{\beta, 1-\alpha\}},
$$

where the Hölder constants $\theta, \tilde{\theta}$ are random variables which depend only on $R, L_{R}, M_{0, T}, M_{0}, L_{0}, T, \alpha, \beta$, $\Lambda_{\alpha}\left(B^{H}\right)$.

We conclude from (3.1) that

$$
\left(\varphi^{\prime}(x) b(t, x), \varphi^{\prime}(x) \sigma(t, x)\right) \in S_{\varphi(K(t))}(t, \varphi(x)) .
$$

We can also give the extension of the result concerning stochastic tangent sets to inverse images to the fBM form.

We introduce a space $\mathcal{H}$ of the functions $\varphi: \mathbb{R}^{d} \rightarrow \mathbb{R}^{m}$ of class $C^{2}$, with a bounded and Lipschitz continuous second derivative and moreover there exist $a_{\varphi}<b_{\varphi}$ and $M>0, L>0$, such that for all $a_{\varphi} \leq|x| \leq b_{\varphi}$, the matrix $\varphi^{\prime}(x)=\frac{\mathrm{d}}{\mathrm{d} x} \varphi(x)=\nabla_{x} \varphi(x) \in \mathbb{R}^{m \times d}$ has a right inverse denoted by $\varphi^{\prime}(x)^{+}$satisfying

$$
\begin{aligned}
& \text { (1) }\left|\left[\varphi^{\prime}(x)^{+}\right]^{\prime}\right| \leq M, \\
& \text { (2) }\left|\left[\varphi^{\prime}(x)^{+}\right]^{\prime}-\left[\varphi^{\prime}(y)^{+}\right]^{\prime}\right| \leq L|x-y|,
\end{aligned}
$$

where we use the notations $\left[\varphi^{\prime}(x)^{+}\right]^{\prime}=\frac{\mathrm{d}}{\mathrm{d} x}\left[\left(\frac{\mathrm{d}}{\mathrm{d} x} \varphi(x)\right)^{+}\right]=\nabla_{x}\left[\left(\nabla_{x} \varphi(x)\right)^{+}\right]$.

Lemma 3.4. Given two stochastic processes $U=U^{t, x}, V=V^{t, x}$ satisfying (2.5), (2.6) with $d=m$, and $\varphi \in \mathcal{H}$, let

$$
\begin{aligned}
& f(r, y)=\varphi^{\prime}(y)^{+}\left[U(r)-\left(\varphi^{\prime}(y)-\varphi^{\prime}(x)\right) b(t, x)\right], \\
& g(r, y)=\varphi^{\prime}(y)^{+}\left[V(r)-\left(\varphi^{\prime}(y)-\varphi^{\prime}(x)\right) \sigma(t, x)\right] .
\end{aligned}
$$


Assume that $\left(\mathbf{H}_{1}\right)$ and $\left(\mathbf{H}_{2}\right)$ are satisfied with $1 / 2<H<1,1-H<\beta, \delta>\frac{1-H}{H}$, then for $\alpha \in$ $\left(1-H, \alpha_{0}\right)$ and for every $\delta_{0}>0$, there exists a random variable $\bar{h}_{1}=\bar{h}_{1}^{t, x}$, such that for $a_{\varphi}+2 \delta_{0}$ $\leq|x| \leq b_{\varphi}-2 \delta_{0}$ and $\mathbb{P}$-a.s. $\omega \in \Omega$, the following stochastic differential equation

$$
\xi_{s}=x+\int_{t}^{s}\left(b(t, x)+f\left(r, \xi_{r}\right)\right) \mathrm{d} r+\int_{t}^{s}\left(\sigma(t, x)+g\left(r, \xi_{r}\right)\right) \mathrm{d} B^{H}(r), s \in\left[t, t+\bar{h}_{1}\right]
$$

has a unique solution $\xi .(\omega) \in L^{0}\left(\Omega, \mathcal{F}, \mathbb{P} ; W^{\alpha, \infty}\left(t, T ; \mathbb{R}^{d}\right)\right)$. Moreover $\xi$. $(\omega) \in C^{1-\alpha}\left(t, t+\bar{h}_{1} ; \mathbb{R}^{d}\right)$, $\mathbb{P}$-a.s.

Proof. Applying the partition of unity theorem from [12], page 61, we see that for every $\delta_{0}>0$, there exists a function $\alpha(x) \in C^{\infty}\left(\mathbb{R}^{d}\right)$ such that $\alpha(x)=1$ for $a_{\varphi}+\delta_{0} \leq|x| \leq b_{\varphi}-\delta_{0}$ and $\alpha(x)=0$ for $|x| \geq b_{\varphi}$ or $|x| \leq a_{\varphi}$, then we define

$$
\tilde{f}(t, y)=\alpha(y) f(t, y)= \begin{cases}f(t, y), & a_{\varphi}+\delta_{0} \leq|y| \leq b_{\varphi}-\delta_{0} \\ \alpha(y) f(t, y) & a_{\varphi} \leq|y| \leq a_{\varphi}+\delta_{0}, \quad \text { or } b_{\varphi}-\delta_{0} \leq|y| \leq b_{\varphi} \\ 0, & |y| \geq b_{\varphi}, \text { or }|y| \leq a_{\varphi} .\end{cases}
$$

We define $\tilde{g}(t, y)$ by the same method, then we consider the following equation

$$
\tilde{\xi}_{s}=x+\int_{t}^{s}\left(b(t, x)+\tilde{f}\left(r, \tilde{\xi}_{r}\right)\right) \mathrm{d} r+\int_{t}^{s}\left(\sigma(t, x)+\tilde{g}\left(r, \tilde{\xi}_{r}\right)\right) \mathrm{d} B^{H}(r), s \in[t, t+\bar{h}] .
$$

Since $\varphi \in \mathcal{H}$ and $U=U^{t, x}, V=V^{t, x}$ satisfy (2.5), (2.6) with $d=m$, we can verify that for $\alpha \in\left(1-H, \alpha_{0}\right)$, $\tilde{f}(t, y)$, and $\tilde{g}(t, y)$ satisfy the conditions of $\left(\mathbf{H}_{1}\right),\left(\mathbf{H}_{2}\right)$ from [11], where $M_{0}, M_{R}, L_{0}, L_{R}$ depend on $\omega$. Hence for all $\alpha \in\left(1-H, \alpha_{0}\right)$, equation (3.2) has a unique solution $\tilde{\xi}$. $(\omega) \in L^{0}\left(\Omega, \mathcal{F}, \mathbb{P} ; W^{\alpha, \infty}\left(t, T ; \mathbb{R}^{d}\right)\right)$ and moreover $\tilde{\xi} .(\omega) \in C^{1-\alpha}\left(t, t+\bar{h} ; \mathbb{R}^{d}\right), \mathbb{P}$-a.s. Since $a_{\varphi}+2 \delta_{0} \leq|x| \leq b_{\varphi}-2 \delta_{0}$, there exists a random variable $\bar{h}_{1}=\bar{h}_{1}^{t, x}$ such that $a_{\varphi}+\delta_{0} \leq|\tilde{\xi}| \leq b_{\varphi}-\delta_{0}, \mathbb{P}$-a.s. Then for $s \in\left[t, t+\bar{h}_{1}\right]$, (3.2) becomes to

$$
\tilde{\xi}_{s}=x+\int_{t}^{s}\left(b(t, x)+f\left(r, \tilde{\xi}_{r}\right)\right) \mathrm{d} r+\int_{t}^{s}\left(\sigma(t, x)+g\left(r, \tilde{\xi}_{r}\right)\right) \mathrm{d} B^{H}(r), s \in\left[t, t+\bar{h}_{1}\right], \mathbb{P}-\text { a.s. }
$$

Taking $\xi_{s}=\tilde{\xi}_{s}, s \in\left[t, t+\bar{h}_{1}\right]$, together with the uniqueness of $\tilde{\xi}_{s}$, the proof is completed.

Theorem 3.5. Assume that $\left(\mathbf{H}_{1}\right)$ and $\left(\mathbf{H}_{2}\right)$ are satisfied with $1 / 2<H<1,1-H<\beta, \delta>\frac{1-H}{H}$. Set $\max \{1-H, 1-\mu\}<\alpha<\alpha_{0}$. Let $K(t)=\overline{K(t)} \subset \mathbb{R}^{d}, t \in[0, T]$ and $\varphi \in \mathcal{H}$. Then for all $x \in \mathbb{R}^{d}$ with $a_{\varphi}<|x|<b_{\varphi}$

$$
(b(t, x), \sigma(t, x)) \in S_{\varphi^{-1}(\varphi(K(t)))}(t, x)
$$

if and only if

$$
\left(\varphi^{\prime}(x) b(t, x), \varphi^{\prime}(x) \sigma(t, x)\right) \in S_{\varphi(K(t))}(t, \varphi(x)) .
$$

Proof. It is sufficient to prove that if $\left(\varphi^{\prime}(x) b(t, x), \varphi^{\prime}(x) \sigma(t, x)\right) \in S_{\varphi(K(t))}(t, \varphi(x))$, then $(b(t, x), \sigma(t, x)) \in$ $S_{\varphi^{-1}(\varphi(K(t)))}(t, x)$.

Since

$$
\left(\varphi^{\prime}(x) b(t, x), \varphi^{\prime}(x) \sigma(t, x)\right) \in S_{\varphi(K(t))}(t, \varphi(x)),
$$

for $x \in K(t)$, there exist a random variable $\bar{h}=\bar{h}^{t, x}>0$ and two stochastic processes $U_{1}$ and $V_{1}$ satisfying (2.5), (2.6) with $d=m$, and for all $s \in[t, t+\bar{h}]$, for every $R>0$ with $|x| \leq R$,

$$
\varphi(x)+\int_{t}^{s}\left(\varphi^{\prime}(x) b(t, x)+U_{1}(r)\right) \mathrm{d} r+\int_{t}^{s}\left(\varphi^{\prime}(x) \sigma(t, x)+V_{1}(r)\right) \mathrm{d} B^{H}(r) \in \varphi(K(s)) .
$$


Let

$$
\begin{aligned}
& f(r, y)=\varphi^{\prime}(y)^{+}\left[U_{1}(r)-\left(\varphi^{\prime}(y)-\varphi^{\prime}(x)\right) b(t, x)\right], \\
& g(r, y)=\varphi^{\prime}(y)^{+}\left[V_{1}(r)-\left(\varphi^{\prime}(y)-\varphi^{\prime}(x)\right) \sigma(t, x)\right],
\end{aligned}
$$

where $\varphi^{\prime}(y)^{+}$is the right inverse of $\varphi^{\prime}(y)$. By Lemma 3.4, for every $\delta_{0}>0$ and $a_{\varphi}+2 \delta_{0} \leq|x| \leq b_{\varphi}-2 \delta_{0}$, there exists a random variable $\bar{h}_{1}$ such that for $\mathbb{P}$-a.s. $\omega \in \Omega$, the following SDE

$$
\xi_{s}=x+\int_{t}^{s}\left(b(t, x)+f\left(r, \xi_{r}\right)\right) \mathrm{d} r+\int_{t}^{s}\left(\sigma(t, x)+g\left(r, \xi_{r}\right)\right) \mathrm{d} B^{H}(r), s \in\left[t, t+\bar{h}_{1}\right]
$$

has a unique solution $\xi$. $(\omega)$. Taking

$$
\begin{aligned}
& U(r)=\varphi^{\prime}\left(\xi_{r}\right)^{+}\left[U_{1}(r)-\left(\varphi^{\prime}\left(\xi_{r}\right)-\varphi^{\prime}(x)\right) b(t, x)\right], \\
& V(r)=\varphi^{\prime}\left(\xi_{r}\right)^{+}\left[V_{1}(r)-\left(\varphi^{\prime}\left(\xi_{r}\right)-\varphi^{\prime}(x)\right) \sigma(t, x)\right],
\end{aligned}
$$

for $r \in\left[t, t+\bar{h}_{1}\right]$ and $U(r)=U\left(t+\bar{h}_{1}\right), V(r)=V\left(t+\bar{h}_{1}\right)$ for $r \in\left[t+\bar{h}_{1}, T\right]$. We deduce that

$$
U(t)=0, \quad V(t)=0 .
$$

Since $\varphi \in \mathcal{H}$ and $\xi .(\omega) \in C^{1-\alpha}\left(t, t+\bar{h}_{1} ; \mathbb{R}^{d}\right)$, from $\left(\mathbf{H}_{1}\right)$ and $\left(\mathbf{H}_{2}\right)$, it follows that

$$
|U(s)-U(\tau)| \leq \theta|s-\tau|^{1-\alpha}, \quad|V(s)-V(\tau)| \leq \tilde{\theta}|s-\tau|^{\min \{\beta, 1-\alpha\}}, \quad \forall s, \tau \in\left[t, t+\bar{h}_{1}\right] .
$$

According to the fractional Itô formula, we know for all $s \in\left[t, t+\bar{h}_{1}\right]$ that

$$
\begin{aligned}
& \varphi\left(x+\int_{t}^{s}(b(t, x)+U(r)) \mathrm{d} r+\int_{t}^{s}(\sigma(t, x)+V(r)) \mathrm{d} B^{H}(r)\right) \\
= & \varphi(x)+\int_{t}^{s}\left(\varphi^{\prime}(x) b(t, x)+U_{1}(r)\right) \mathrm{d} r+\int_{t}^{s}\left(\varphi^{\prime}(x) \sigma(t, x)+V_{1}(r)\right) \mathrm{d} B^{H}(r) \in \varphi(K(s)) .
\end{aligned}
$$

which means that

$$
(b(t, x), \sigma(t, x)) \in S_{\varphi^{-1}(\varphi(K(t)))}(t, x) .
$$

\section{Applications to Deterministic Characterization of Viability}

Using Theorem 3.5, if $K$ takes some particular forms and $B^{H}$ is one-dimensional, we will get deterministic sufficient and necessary conditions for viability which can be checked more easily. Firstly we prove some preliminary results.

Lemma 4.1. Assume that $\left(\mathbf{H}_{1}\right)$ and $\left(\mathbf{H}_{2}\right)$ are satisfied with $k=1,1 / 2<H<1,1-H<\beta, \delta>\frac{1-H}{H}$. Let $\max \{1-H, 1-\mu\}<\alpha<\alpha_{0}$ and $K=\left\{x \in \mathbb{R}^{d} ; r \leq|x| \leq R\right\}$. Then for all $x$, such that $|x|=R$,

$$
(b(t, x), \sigma(t, x)) \in S_{K}(t, x) \text { if and only if }\langle x, b(t, x)\rangle \leq 0,\langle x, \sigma(t, x)\rangle=0,
$$

and for all $x$, such that $|x|=r$,

$$
(b(t, x), \sigma(t, x)) \in S_{K}(t, x) \text { if and only if }\langle x, b(t, x)\rangle \geq 0,\langle x, \sigma(t, x)\rangle=0 .
$$

Proof. Taking $\varphi(x)=|x|^{2}$, for $R / 4 \leq|x| \leq 4 R$, we have

$$
\varphi^{\prime}(x)^{+}=\frac{x}{2|x|^{2}} .
$$


We can verify that $\varphi \in \mathcal{H}$ with $a_{\varphi}=R / 4, b_{\varphi}=4 R$. For $|x|=R$, we know that $a_{\varphi}<|x|<b_{\varphi}$. We have also similar discussion with respect to $|x|=r$. By Theorem 3.5, Lemma 4.1 is reduced to the following equivalence:

For all $x$ such that $|x|=R$

$$
(\langle 2 x, b(t, x)\rangle,\langle 2 x, \sigma(t, x)\rangle) \in S_{\varphi(K)}\left(t,|x|^{2}\right) \Leftrightarrow\langle x, b(t, x)\rangle \leq 0,\langle x, \sigma(t, x)\rangle=0 .
$$

For all $x$, such that $|x|=r$,

$$
(\langle 2 x, b(t, x)\rangle,\langle 2 x, \sigma(t, x)\rangle) \in S_{\varphi(K)}\left(t,|x|^{2}\right) \Leftrightarrow\langle x, b(t, x)\rangle \geq 0,\langle x, \sigma(t, x)\rangle=0 .
$$

It's convenient to prove only the case: $|x|=R$, the other one is similar.

Necessary. If $\langle x, b(t, x)\rangle \leq 0,\langle x, \sigma(t, x)\rangle=0$, taking $U(r) \equiv 0, V(r) \equiv 0$, we can choose $\bar{h}$ small enough, such that $\forall s \in[t, t+\bar{h}]$,

$$
r^{2} \leq|x|^{2}+\int_{t}^{s}(\langle 2 x, b(t, x)\rangle+U(y)) \mathrm{d} y+\int_{t}^{s}(\langle 2 x, \sigma(t, x)\rangle+V(y)) \mathrm{d} B^{H}(y) \leq R^{2} .
$$

This means that $(\langle 2 x, b(t, x)\rangle,\langle 2 x, \sigma(t, x)\rangle) \in S_{\varphi(K)}\left(t,|x|^{2}\right)$.

Sufficient. Since $(\langle 2 x, b(t, x)\rangle,\langle 2 x, \sigma(t, x)\rangle) \in S_{\varphi(K)}\left(t,|x|^{2}\right)$, there exist a random variable $\bar{h}=$ $\bar{h}^{t, x}>0$, and two stochastic processes satisfying (2.5), (2.6) with $d=1, k=1$, and for all $s \in[t, t+\bar{h}]$, for $|x| \leq R$,

$$
r^{2} \leq|x|^{2}+\int_{t}^{s}(\langle 2 x, b(t, x)\rangle+U(y)) \mathrm{d} y+\int_{t}^{s}(\langle 2 x, \sigma(t, x)\rangle+V(y)) \mathrm{d} B^{H}(y) \leq R^{2} .
$$

Since $|x|=R$, it follows that

$$
\langle 2 x, b(t, x)\rangle(s-t)+\langle 2 x, \sigma(t, x)\rangle\left(B^{H}(s)-B^{H}(t)\right)+\int_{t}^{s} U(y) \mathrm{d} y+\int_{t}^{s} V(y) \mathrm{d} B^{H}(y) \leq 0 .
$$

By (2.3), there exists $\Omega_{0} \subset \Omega$ with $\mathbb{P}\left(\Omega_{0}\right)=1 / 2$, such that for each $\omega_{0} \in \Omega_{0}$, (4.1) is satisfied and there is a sequence $t \leq s_{n}=s_{n}\left(\omega_{0}\right) \leq t+\bar{h}\left(\omega_{0}\right), s_{n} \searrow t$, such that

$$
\lim _{s_{n} \searrow t} \frac{B_{s_{n}}^{H}\left(\omega_{0}\right)-B_{t}^{H}\left(\omega_{0}\right)}{s_{n}-t}=+\infty
$$

Then we conclude that

$$
\langle 2 x, \sigma(t, x)\rangle \frac{B_{\omega_{0}}^{H}\left(s_{n}\right)-B_{\omega_{0}}^{H}(t)}{s_{n}-t}+\left[\int_{t}^{s_{n}} U(y) \mathrm{d} y+\int_{t}^{s_{n}} V(y) \mathrm{d} B_{\omega_{0}}^{H}(y)\right] \frac{1}{s_{n}-t} \leq-\langle 2 x, b(t, x)\rangle .
$$

By Lemma 3.1

$$
\left[\int_{t}^{s_{n}} U(y) \mathrm{d} y+\int_{t}^{s_{n}} V(y) \mathrm{d} B_{\omega_{0}}^{H}(y)\right] \frac{1}{s_{n}-t} \rightarrow 0
$$

recalling (4.2), yields

$$
\langle x, \sigma(t, x)\rangle \leq 0
$$

Similarly we can prove that $\langle x, \sigma(t, x)\rangle \geq 0$, choosing $\omega_{0}^{\prime}$ and a sequence $t \leq r_{n}=r_{n}\left(\omega_{0}^{\prime}\right) \leq t+\bar{h}\left(\omega_{0}^{\prime}\right), r_{n} \searrow t$, such that $\lim _{r_{n} \searrow t} \frac{B_{r_{n}}^{H}\left(\omega_{0}^{\prime}\right)-B_{t}^{H}\left(\omega_{0}^{\prime}\right)}{r_{n}-t}=-\infty$. Consequently,

$$
\langle x, \sigma(t, x)\rangle=0 .
$$


Then from (4.3), we deduce that

$$
\langle 2 x, b(t, x)\rangle+\left[\int_{t}^{s} U(y) \mathrm{d} y+\int_{t}^{s} V(y) \mathrm{d} B^{H}(y)\right] \frac{1}{s-t} \leq 0 .
$$

Let $s \rightarrow t$. Via Lemma 3.1, it follows that

$$
\langle x, b(t, x)\rangle \leq 0
$$

As particular cases, we have the following two corollaries

Corollary 4.2. Assume that $\left(\mathbf{H}_{1}\right)$ and $\left(\mathbf{H}_{2}\right)$ are satisfied with $k=1,1 / 2<H<1,1-H<\beta, \delta>\frac{1-H}{H}$. Let $\max \{1-H, 1-\mu\}<\alpha<\alpha_{0}$ and $K=\left\{x \in \mathbb{R}^{d} ;|x|=1\right\}$ (the unit sphere). Then for all $x \in K$,

$$
(b(t, x), \sigma(t, x)) \in S_{K}(t, x) \text { if and only if }\langle x, b(t, x)\rangle=0,\langle x, \sigma(t, x)\rangle=0 .
$$

Corollary 4.3. Assume that $\left(\mathbf{H}_{1}\right)$ and $\left(\mathbf{H}_{2}\right)$ are satisfied with $k=1,1 / 2<H<1,1-H<\beta, \delta>\frac{1-H}{H}$. Let $\max \{1-H, 1-\mu\}<\alpha<\alpha_{0}$ and $K=\left\{x \in \mathbb{R}^{d} ;|x| \leq 1\right\}$ (the unit ball). Then for all $x$, such that $|x|=1$,

$$
(b(t, x), \sigma(t, x)) \in S_{K}(t, x) \text { if and only if }\langle x, b(t, x)\rangle \leq 0,\langle x, \sigma(t, x)\rangle=0 .
$$

As it is shown in Corollary 2.12, if we want to get conditions for the viability of $K$, we only need to consider the starting point $x \in \partial K$. Then, from Corollaries 4.2 and 4.3, we have respectively the following two results.

Proposition 4.4. Let $\left(\mathbf{H}_{1}\right),\left(\mathbf{H}_{2}\right)$ be satisfied with $k=1,1 / 2<H<1,1-H<\beta, \delta>\frac{1-H}{H}$. Let $\max \{1-H, 1-\mu\}<\alpha<\alpha_{0}$ and $K$ be the unit sphere. Then, the following assertions are equivalent:

(I) $K$ is viable for the fractional SDE (2.4);

(II) for all $t \in[0, T]$ and all $x \in K$,

$$
\langle x, b(t, x)\rangle=0,\langle x, \sigma(t, x)\rangle=0 .
$$

Proposition 4.5. Let $\left(\mathbf{H}_{1}\right),\left(\mathbf{H}_{2}\right)$ be satisfied with $k=1,1 / 2<H<1,1-H<\beta, \delta>\frac{1-H}{H}$. Let $\max \{1-H, 1-\mu\}<\alpha<\alpha_{0}$ and $K$ be the unit ball. Then the following assertions are equivalent:

(I) $K$ is viable for the fractional SDE (2.4);

(II) for all $t \in[0, T]$ and all $|x|=1$,

$$
\langle x, b(t, x)\rangle \leq 0,\langle x, \sigma(t, x)\rangle=0 .
$$

As a consequence of Theorem 3.5, we have the following

Corollary 4.6. Considering the one-dimensional SDE,

$$
X_{s}=x+\int_{t}^{s} b\left(r, X_{r}\right) \mathrm{d} r+\int_{t}^{s} \sigma\left(r, X_{r}\right) \mathrm{d} B_{r}^{H}, s \in[t, T] .
$$

Suppose that $\left(\mathbf{H}_{1}\right)$ and $\left(\mathbf{H}_{2}\right)$ are satisfied with $k=1$. Then, for any $t \in[0, T]$ and every $x \geq 0$, the above equation has a positive solution if and only if

$$
b(t, 0) \geq 0, \sigma(t, 0)=0, \quad \forall t \in[0, T] .
$$

Proof. Taking $\varphi(x)=x$ and $K=[0,+\infty)$. Analysis similar to that in the proof of Lemma 4.1 shows that $(b(t, 0), \sigma(t, 0)) \in S_{K}(t, 0)$ if and only if $b(t, 0) \geq 0, \sigma(t, 0)=0$. Considering Corollary 2.12, we get our result.

Another interesting application is the comparison of the solution theorem. 


\section{Comparison theorem}

Corollary 5.1. Considering the linear two-dimensional decoupled system,

$$
\left\{\begin{array}{l}
X_{s}^{t, x}=x+\int_{t}^{s}\left(f(r) X_{r}^{t, x}+f_{1}(r)\right) \mathrm{d} r+\int_{t}^{s}\left(g(r) X_{r}^{t, x}+g_{1}(r)\right) \mathrm{d} B_{r}^{H}, s \in[t, T] \\
Y_{s}^{t, y}=y+\int_{t}^{s}\left(f(r) Y_{r}^{t, y}+f_{2}(r)\right) \mathrm{d} r+\int_{t}^{s}\left(g(r) Y_{r}^{t, y}+g_{2}(r)\right) \mathrm{d} B_{r}^{H}, s \in[t, T]
\end{array}\right.
$$

where $B^{H}$ is one-dimensional, $f(r), f_{1}(r), g(r), g_{1}(r)$ are Lipschitz functions.

Then the two assertions are equivalent:

- $\quad$ for any $t \in[0, T]$ and every $x \leq y: X_{s}^{t, x} \leq Y_{s}^{t, y}, \forall s \in[t, T]$;

- $f_{1}(t) \leq f_{2}(t), g_{1}(t)=g_{2}(t), \forall t \in[0, T]$.

Proof. Setting $Z_{s}^{t, z}=Y_{s}^{t, y}-X_{s}^{t, x}, z=y-x \geq 0$, we can change our problem to an equivalent form:

$$
\begin{gathered}
\text { for any } t \in[0, T] \text { and every } z \geq 0, \text { the solution } Z_{s}^{t, z} \geq 0 \\
\Longleftrightarrow f_{1}(t) \leq f_{2}(t), g_{1}(t)=g_{2}(t), \forall t \in[0, T] .
\end{gathered}
$$

Using Corollary 4.6, the proof is completed.

In general case, we can prove

Theorem 5.2 (comparison theorem). Considering the two-dimensional decoupled system

$$
\left\{\begin{array}{l}
X_{s}^{t, x}=x+\int_{t}^{s}\left(b_{1}\left(r, X_{r}^{t, x}\right)\right) \mathrm{d} r+\int_{t}^{s}\left(\sigma_{1}\left(r, X_{r}^{t, x}\right)\right) \mathrm{d} B_{r}^{H}, \quad s \in[t, T], \\
Y_{s}^{t, y}=y+\int_{t}^{s}\left(b_{2}\left(r, Y_{r}^{t, y}\right)\right) \mathrm{d} r+\int_{t}^{s}\left(\sigma_{2}\left(r, Y_{r}^{t, y}\right)\right) \mathrm{d} B_{r}^{H}, \quad s \in[t, T],
\end{array}\right.
$$

where $\left(\mathbf{H}_{1}\right)$ and $\left(\mathbf{H}_{2}\right)$ are satisfied with $k=1,1 / 2<H<1,1-H<\beta, \delta>\frac{1-H}{H}$. Let $\max \{1-H, 1-\mu\}<$ $\alpha<\alpha_{0}$, then the two assertions are equivalent:

- $\quad$ for any $t \in[0, T]$ and every $x \leq y: X_{s}^{t, x} \leq Y_{s}^{t, y}, \forall s \in[t, T]$;

- $b_{1}(t, z) \leq b_{2}(t, z), \sigma_{1}(t, z)=\sigma_{2}(t, z), \forall t \in[0, T], \forall z \in \mathbb{R}$.

Proof. We write the two-dimensional decoupled system in the following form

$$
Z_{s}^{t, z}=z+\int_{t}^{s}\left(b\left(r, Z_{r}^{t, z}\right)\right) \mathrm{d} r+\int_{t}^{s}\left(\sigma\left(r, Z_{r}^{t, z}\right)\right) \mathrm{d} B_{r}^{H}, s \in[t, T]
$$

where

$$
Z_{s}^{t, z}=\left(\begin{array}{c}
X_{s}^{t, x} \\
Y_{s}^{t, y}
\end{array}\right), z=\left(\begin{array}{c}
x \\
y
\end{array}\right), b\left(r, Z_{r}^{t, z}\right)=\left(\begin{array}{l}
b_{1}\left(r, X_{r}^{t, x}\right) \\
b_{2}\left(r, Y_{r}^{t, y}\right)
\end{array}\right), \sigma\left(r, Z_{r}^{t, z}\right)=\left(\begin{array}{l}
\sigma_{1}\left(r, X_{r}^{t, x}\right) \\
\sigma_{2}\left(r, Y_{r}^{t, y}\right)
\end{array}\right) .
$$

Taking $\varphi(z)=\varphi(x, y)=y-x$, then for every $z \in \mathbb{R}^{2}, \varphi^{\prime}(z)=(-1,1)$, and

$$
\varphi^{\prime}(z)^{+}=\frac{1}{2}\left(\begin{array}{c}
-1 \\
1
\end{array}\right)
$$

Consequently $\varphi \in \mathcal{H}$. Setting $K=\left\{\left(\begin{array}{l}x \\ y\end{array}\right) \mid y-x \geq 0\right\}$, we know that $\varphi(K)=\mathbb{R}^{+}$and $\varphi^{-1}(\varphi(K))=K$. By Theorem 3.5 and Corollary 2.12, the viability of $K$ is equivalent to

$$
\left(\varphi^{\prime}(z) b(t, z), \varphi^{\prime}(z) \sigma(t, z)\right) \in S_{\mathbb{R}^{+}}(t, \varphi(z)), \forall t \in[0, T], \forall z=\left(\begin{array}{l}
x \\
y
\end{array}\right) \in \partial K=\left\{\left(\begin{array}{l}
x \\
y
\end{array}\right): x=y\right\},
$$


that is

$$
\left(b_{2}(t, x)-b_{1}(t, x), \sigma_{2}(t, x)-\sigma_{1}(t, x)\right) \in S_{\mathbb{R}^{+}}(t, 0) .
$$

Now following the same calculus as in Lemma 4.1, we have

$$
\begin{aligned}
S_{\mathbb{R}^{+}}(t, 0)= & \left\{(u, v) \in \mathbb{R}^{2}: u(s-t)+v\left(B_{s}^{H}-B_{t}^{H}\right)\right. \\
& \left.+\int_{t}^{s} U(y) \mathrm{d} y+\int_{t}^{s} V(y) \mathrm{d} B_{y}^{H} \geq 0, \quad \forall s \in[t, t+\bar{h}]\right\} \\
= & \left\{(u, 0) \in \mathbb{R}^{2}: u \geq 0\right\},
\end{aligned}
$$

which complete the proof.

Remark 5.3. We can consider the following control problem: find two control stochastic processes $u$ and $v$ such that the state $X$ driven by the system

$$
X_{s}=X_{0}+\int_{0}^{s}\left[b\left(r, X_{r}\right)+u(r)\right] \mathrm{d} r+\int_{0}^{s}\left[\sigma\left(r, X_{r}\right)+v(r)\right] \mathrm{d} B_{r}^{H}, \quad s \in[0, T],
$$

evolves in given constraint closed set $K$. We ask moreover that the controls are in some feedback forms. As an example:

Let $K=\left\{x \in \mathbb{R}^{d}:|x| \leq R\right\}$, where $R$ is a fixed positive constant, and $\Pi_{K}: \mathbb{R}^{d} \rightarrow K, \Pi_{K}(x)=\frac{x}{|x|}(|x| \wedge R)$ the projector operator on $K$. Find bounded feedback control processes $u$ and $v$ of the form $u(t)=\alpha \Pi_{K}(X(t))$ and $v(t)=g(t, X(t)) \Pi_{K}(X(t))$ such that

$$
\left|X_{s}^{x, u, v}\right| \leq R, \quad \forall s \in[0, T], \quad \forall|x| \leq R .
$$

By Corollary 4.3 such controls exist taking

$$
\alpha \leq-\frac{1}{R^{2}} \sup _{|x|=R, t \in[0, T]}\langle x, b(t, x)\rangle \quad \text { and } \quad g(t, x)=-\frac{1}{R^{2}}\left\langle\Pi_{K}(x), \sigma\left(t, \Pi_{K}(x)\right)\right\rangle .
$$

Acknowledgements. The authors wish to expresses their thanks to Rainer Buckdahn and Lucian Maticiuc for their useful suggestions and discussions. The authors also thank the referees for their helpful comments and suggestions.

\section{REFERENCES}

[1] J.P. Aubin and G. Da Prato, Stochastic viability and invariance. Ann. Scuola Norm. Super. Pisa Cl. Sci. 27 (1990) 595-694.

[2] F. Biagini, Y. Hu, B. Øksendal and T. Zhang, Stochastic calculus for fractional Brownian motion and applications. Springer (2006).

[3] R. Buckdahn, M. Quincampoix and A. Rascanu, Propriété de viabilité pour des équations différentielles stochastiques rétrogrades et applications à des équations aux derivées partielles. C. R. Acad. Sci. Paris Sér. I 325 (1997) 1159-1162.

[4] R. Buckdahn, S. Peng, M. Quincampoix and C. Rainer, Existence of stochastic control under state constraints. C. R. Acad. Sci. Paris Sér. I 327 (1998) 17-22.

[5] R. Buckdahn, M. Quincampoix and A. Rascanu, Viability property for backward stochastic differential equation and applications to partial differential equation. Probab. Theory Relat. Fields 116 (2000) 485-504.

[6] R. Buckdahn, M. Quincampoix, C. Rainer and A. Rascanu, Viability of moving sets for stochastic differential equation. Adv. Differential Equations 7 (2002) 1045-1072.

[7] I. Ciotir and A. Rascanu, Viability for stochastic differential equation driven by fractional Brownian motions. J. Differential Equations 247 (2009) 1505-1528.

[8] B.B. Mandelbrot and J.W. Van Ness, Fractional Brownian motions, fractional noises and applications. SIAM Rev. 10 (1968) $422-437$.

[9] A. Milian, A note on stochastic invariance for Ito equations. Bull. Pol. Acad. Sci., Math. 41 (1993) 139-150.

[10] Y.S. Mishura, Stochastic calculus for fractional Brownian motion and related processes. Springer (2007).

[11] D. Nualart and A. Rascanu, Differential equations driven by fractional Brownian motion. Collect. Math. 53 (2002) 55-81.

[12] K. Yosida, Functional Analysis. Springer (1971). 